\title{
REGULAR PARTIAL DIFFERENTIAL EQUATIONS
}

\section{GARRETT BIRKHOFF AND THOMAS MULLIKIN ${ }^{1}$}

1. Introduction. We consider below the Cauchy problem for systems of homogeneous linear partial differential equations ( $D E$ 's) with constant coefficients. That is, we ask for solutions of

$$
\partial u_{j} / \partial t=\sum p_{j k}\left(D_{1}, \cdots, D_{r}\right) u_{k} \quad[j, k=1, \cdots, n],
$$

satisfying $u(x ; 0)=v_{0}(x)$, where $v_{0}(x)$ is supposed given in infinite $\left(x_{1}, \cdots, x_{r}\right)$-space. Here $\left\|p_{j k}(D)\right\|$ denotes a general matrix of polynomials with constant coefficients in the differential operators $D_{i}=\partial / \partial x_{i}$.

Our main result is an existence theorem for the Cauchy problem in infinite space, for any system (1) which is regular in the following sense [1]. For each real wave-vector $q$, we let $\lambda_{1}(q), \cdots, \lambda_{n}(q)$ denote the characteristic values of the $\boldsymbol{q}$-matrix $\left\|p_{j k}(i \boldsymbol{q})\right\|, i=(-1)^{1 / 2}$. We define the spectral norm of (1) as

$$
\sigma[P]=\sup _{\boldsymbol{q}, j} \operatorname{Re}\left\{\lambda_{j}(\boldsymbol{q})\right\}=\sup _{\boldsymbol{q}} \sigma[P(\boldsymbol{q})],
$$

and call the system (1) regular when $\sigma[P]<+\infty$.

In this existence theorem, the solutions are defined as the semiorbits, for $t>0$, of a $C_{0}$-semigroup acting on an appropriate Banach space. Thus, in particular, we solve a family of "abstract Cauchy problems" $([5 ; 6])$, each corresponding in some sense to the system (1). Further, it is shown that the $C_{0}$-semigroup can be so constructed that all semi-orbits represent literal solutions of (1). Finally, in the "hyperbolic" case, a $C_{0}$-group can be constructed.

Though similar methods have been used by Gelfand and Silov $([3 ; 4])$, the representation in terms of $C_{0}$-semigroups is new. The specific results of Hille [5] are for quite special $D E$ 's. Related results have also been obtained by L. Schwartz [7] and, using other methods, by L. Gårding (Acta Math. vol. 85 (1951) pp. 1-62) and A. Lax (Communications on Pure and Applied Mathematics, vol. 9 (1956) pp. 135-70).

2. The Fréchet space $\Phi$. For any fixed wave-vector $q=\left(q_{1}, \cdots, q_{r}\right)$, any system (1) has solutions of the special form

Received by the editors June 26, 1956 and, in revised form, May 17, 1957.

${ }_{1}^{1}$ Mr. Mullikin's work was supported under Contract N5ori-07634 with the Office of Naval Research. 


$$
u_{j}(\mathbf{x} ; t)=f_{j}(\boldsymbol{q} ; t) e^{i \boldsymbol{q} \cdot \mathbf{x}}, \quad \boldsymbol{q} \cdot \mathbf{x}=q_{1} x_{1}+\cdots+q_{r} x_{r} .
$$

Namely, it is sufficient that $\boldsymbol{f}(\boldsymbol{q} ; t)$ satisfy

$$
d f_{j} / d t=\sum p_{j k}(i \boldsymbol{q}) f_{k} \quad[j, k=1, \cdots, n],
$$

which may be regarded as a system of ordinary $D E$ 's because $\boldsymbol{q}$ is fixed.

Now let $\Phi$ denote the vector space of all Borel functions $\boldsymbol{\phi}=\boldsymbol{\phi}(\boldsymbol{q})$, two such functions being identified when they differ on a set of measure zero. If we write formally

$$
v(\mathbf{x})=\int_{Q} e^{i \boldsymbol{q} \cdot \mathbf{x}} \boldsymbol{\phi}(\boldsymbol{q}) d Q, \quad d Q=d q_{1} \cdots d q_{r},
$$

then (1) defines, through $(3)-\left(3^{*}\right)$, the group

$$
T_{t}[\boldsymbol{\phi}(\boldsymbol{q})]=\boldsymbol{\phi}(\boldsymbol{q}) e^{t P(i \boldsymbol{q})}, \quad P(i \boldsymbol{q})=\left\|p_{j k}(i \boldsymbol{q})\right\| .
$$

This is a symbolic solution of (1) in the Fourier transform space $\Phi$ dual to the space of $\boldsymbol{v}(\mathbf{x})$.

Further, we can define $\Phi$ as a Fréchet space, ${ }^{2}$ or topological linear space, by making

$$
\phi_{n} \rightarrow \phi \text { mean } \phi_{n}(q) \rightarrow \phi(q) \text { a.e. }
$$

However, this formal solution is unsatisfactory, as a consideration of the case $u_{t}= \pm u_{x x}$ shows.

3. Norms on $\Phi$. Various norms can be defined on $\Phi$, as follows. Let $C=C(q)$ be any Borel function ${ }^{3}$ from the space $Q$ of real $r$-vectors $q$, to the space of $n \times n$ nonsingular complex matrices $C$. We define

$$
N^{2}(\boldsymbol{q} ; \boldsymbol{\phi})=(\boldsymbol{\phi} C, \boldsymbol{\phi} C)=\boldsymbol{\phi} C(\boldsymbol{q}) C^{\prime *}(\boldsymbol{q}) \boldsymbol{\phi}^{\prime *}
$$

as a complex inner product, and correspondingly

$$
N(\boldsymbol{q} ; \boldsymbol{\phi})=\left[\boldsymbol{\phi} C(\boldsymbol{q}) C^{* *}(\boldsymbol{q}) \boldsymbol{\phi}^{\prime *}\right]^{1 / 2} \geqq 0 .
$$

Since $C$ is nonsingular, $N(\boldsymbol{q} ; \boldsymbol{\phi})=0$ if and only if $\boldsymbol{\phi}=0$ in $\Phi$ (i.e., $\phi_{j}(\boldsymbol{q})=0$ a.e. $)$.

For any such $C(\boldsymbol{q})$, the "norm" $N(\boldsymbol{q}, \boldsymbol{\phi})$ is a non-negative realvalued Borel function on $E_{n} \times Q$ which, for each real $r$-vector $\boldsymbol{q}$, makes the space of complex $n$-vectors $\boldsymbol{\phi}$ into a unitary space $E_{n}(N, \boldsymbol{q})$.

${ }^{2}$ C. Kuratowski, Topologie, first ed., p. 77.

${ }^{3}$ Function "measurable B" in the sense of C. Kuratowski, Topologie, 2d ed, vol. 1, p. 280. We require that the inverse image of a Borel set be a Borel set. Borel functions are measurable, and have the merit of being closed under composition (any Borel function of a Borel function is a Borel function). 
LEMma 1. For any $\boldsymbol{\phi} \in \Phi$, the integral

$$
N(\boldsymbol{\phi})=\int_{Q} N(\boldsymbol{q} ; \boldsymbol{\phi}(\boldsymbol{q})) d Q
$$

is defined, as a non-negative real number or $+\infty$.

Proof. First, $N(\boldsymbol{q} ; \boldsymbol{\phi}(\boldsymbol{q}))$ is a Borel function for all $\boldsymbol{\phi} \in \Phi$. Since $N(\boldsymbol{q} ; \boldsymbol{\phi}) \geqq 0$, the conclusion follows immediately. In this proof, it is essential that the functions involved be Borel functions, and not merely measurable.

Lemma 2. Under the norm (8), the set of $\boldsymbol{\phi} \in \Phi$ with $N(\boldsymbol{\phi})<+\infty$ is a Banach space $B(N)$.

This result is an immediate corollary of standard Lebesgue theory, and the definition of a Banach space. Similar constructions have in fact been used by other authors. ${ }^{4}$

In the subsequent part of this paper, we shall adopt the following notational conventions. $E_{n}$ will denote the unitary space of complex $n$-tuples $\boldsymbol{\phi}=\left(c_{1}, \cdots, c_{n}\right)$ with norm $|\boldsymbol{\phi}|=\left(\sum_{i=1}^{n} c_{i} \bar{c}_{i}\right)^{1 / 2} \cdot E_{n}(N, \boldsymbol{q})$ will denote an $r$-parameter family of unitary spaces of complex $n$ tuples $\boldsymbol{\phi}$ with norms $N(\boldsymbol{q} ; \boldsymbol{\phi}) . B(N)$ will denote the Banach space of complex vector-valued functions $\boldsymbol{\phi}$ with $N(\boldsymbol{\phi})=\int N(\boldsymbol{q} ; \boldsymbol{\phi}) d Q$.

The norm or "modulus" of a linear operator $A(\boldsymbol{q})$ on $E_{n}(N, \boldsymbol{q})$ will be defined by $\|A(\boldsymbol{q})\|_{N}=\sup N(\boldsymbol{q} ; A(\boldsymbol{q})[\boldsymbol{\phi}])$ for $N(\boldsymbol{q} ; \boldsymbol{\phi})=1$. The norm of a bounded linear operator $A$ on $B(N)$ will be defined by $\|A\|_{N}=\sup N(A[\phi])$ for $N(\phi)=1$.

THEOREM 1. For each $\boldsymbol{q}$, let $T_{t}(\boldsymbol{q})$ be a semigroup of linear transformations on the space $E_{n}$, depending continuously on $q$ and $t \geqq 0$. As operators on $E_{n}(N, \boldsymbol{q})$, let the $T_{t}(\boldsymbol{q})$ have uniformly bounded moduli on $0<t<1$. Then the semigroup $\left\{T_{t}\right\}$ on $B(N)$, defined by (5) and (8) for $t>0$, is a $C_{0}$-semigroup.

Proof. Because of the continuous dependence of $T_{t}(\boldsymbol{q})$ on $\boldsymbol{q}$, $T_{t}[\boldsymbol{\phi}]$ is always a Borel function; hence $\left\{T_{t}\right\}$ is always a semigroup on $\Phi$. If, for fixed $t$, the $T_{t}(q)$ have norms bounded by $K(t)<+\infty$ then, substituting (8) into (5), we get

$$
N\left(T_{t}[\boldsymbol{\phi}]\right)=K(t) \int_{Q} N(\boldsymbol{q} ; \boldsymbol{\phi}(\boldsymbol{q})) d Q=K(t)\|\boldsymbol{\phi}\|_{N}
$$

${ }^{4} \mathrm{~F}$. Cunningham, L'structure in Banach spaces, Harvard Doctoral Thesis, 1953. For $p=2$, see J. von Neumann, On rings of operators, Reduction theory, Ann. Math. vol. 50 (1949). 
Hence $T_{t}$ transforms the subspace $B(N)$ of $\Phi$ into itself, and is a linear operator of norm at most $K(t)$ on this Banach space.

It remains [6, p. 18] to show that, for each $\phi \in B(N)$ the orbit $T_{t}[\boldsymbol{\phi}]$ tends continuously to $\boldsymbol{\phi}$ as $t \downarrow 0$-i.e., that

$$
0=\operatorname{Lim}_{t \rightarrow 0^{+}} N\left(T_{t}[\boldsymbol{\phi}]-\boldsymbol{\phi}\right)=\operatorname{Lim}_{t \rightarrow 0^{+}} \int_{Q} N\left(\boldsymbol{q} ; T_{t}[\boldsymbol{\phi}]-\boldsymbol{\phi}\right) d Q .
$$

But now, for any $\phi \in B(N)$,

$$
0 \leqq N\left(\boldsymbol{q} ; T_{t}[\boldsymbol{\phi}(\boldsymbol{q})]-\boldsymbol{\phi}(\boldsymbol{q})\right) \leqq(K(t)+1) N(\boldsymbol{q} ; \boldsymbol{\phi}(\boldsymbol{q}))
$$

where

$$
\int N(\boldsymbol{q} ; \boldsymbol{\phi}(\boldsymbol{q})) d Q=\|\boldsymbol{\phi}\|_{N}<+\infty .
$$

Hence Lebesgue's Dominated Convergence Theorem ${ }^{5}$ applies, and, since $T_{t}[\boldsymbol{\phi}] \rightarrow \boldsymbol{\phi}(\boldsymbol{q})$ for each $\boldsymbol{q}$ (i.e., $N\left(\boldsymbol{q} ; T_{t}[\boldsymbol{\phi}]-\boldsymbol{\phi}\right) \rightarrow 0$ our result is proved.

We note that $T_{t}$ is a direct integral ${ }^{6}$ of operators on the subspaces of $B(N)$ corresponding to Borel subsets of $Q$; by (8) and (9), the norm of each $T_{t}$ is the l.u.b. of the norms of the linear operators

$$
\left\{\exp t\left\|p_{j k}(i q)\right\|\right\}
$$

acting on the $E_{n}(N ; \boldsymbol{q})$.

4. Regular case. We now assume that the system (1) is regular in the sense of (2), that $\sigma[P]<+\infty$. In this case, for each $q \in Q$, we can choose $C(\boldsymbol{q})$ so that, under the norm $\left(7^{\prime}\right)$, the modulus of $\left\{T_{t}(\boldsymbol{q})\right\}$ exceeds $e^{\sigma[P] t}$ by arbitrarily little.

Lemma 3. Given $q \in Q$ and $\eta>0$, we can so choose $C(q)$ that, on $E_{n}(N ; \boldsymbol{q})$,

$$
\left\|T_{t}(\boldsymbol{q})\right\|_{N} \leqq \exp \{(\sigma[P(\boldsymbol{q})]+\eta) t\}, \quad t>0 .
$$

Proof. A slight modification of standard ${ }^{7}$ arguments shows that there always exists a matrix $C(\boldsymbol{q} ; \eta)$ for $P(i \boldsymbol{q})$, such that

$$
C^{-1} P(i \boldsymbol{q}) C=J(\boldsymbol{q} ; \eta)
$$

has entries $\lambda_{j}(q)$ on the main diagonal, zeros and $\eta$ 's just above this diagonal, and all other entries zero. For any such $C(q ; \eta)$, the modulus $\left\|T_{t}(q)\right\|_{N}$ of $T_{t}(q)$ for the norm $\left(7^{\prime}\right)$ is that of $e^{J t}$ operating on the

${ }^{5}$ For this result, see E. J. McShane, Integration, Princeton, 1944, p. 168.

${ }^{6}$ L. Loomis, An introduction to abstract harmonic analysis, New York, 1953, p. 176.

${ }^{7}$ See for example G. Birkhoff and S. MacLane, A survey of modern algebra, rev. ed., Ch. X. 
unitary space of complex $n$-vectors $\psi$, under the ordinary norm $\left(\psi \psi^{\prime *}\right)^{1 / 2}=|\psi|$. But an explicit calculation shows that this is at most $\exp \{(\sigma[P(\boldsymbol{q})]+\eta) t\}$.

Corollary. Let $\boldsymbol{q}_{0} \in Q$ be given, and let $\sigma>\sigma\left(P\left[\boldsymbol{q}_{0}\right]\right)$. Then $C=C\left(\boldsymbol{q}_{0}\right)$ exists such that, throughout some neighborhood $R\left(\boldsymbol{q}_{0}\right)$ of $\boldsymbol{q}_{0}$,

$$
\left\|T_{t}(q)\right\|_{N} \leqq e^{\sigma t} \quad \text { for all } t>0 .
$$

This follows from Lemma 3 , because of the continuity of the modulus of $\left\|T_{t}(\boldsymbol{q})\right\|_{N}$, as a function of $\boldsymbol{q}$, for any fixed $C_{0}=C\left(\boldsymbol{q}_{0}\right)$.

5. Abstract Cauchy problem. The preceding results lead directly to a solution of the abstract Cauchy problem, for any regular system (1). We first prove

Theorem 2. Let (1) be regular, and let $\sigma>\sigma(q)$ for all $q \in Q$. Then a Borel function $C(\boldsymbol{q})$ exists, such that (12) holds for all $\boldsymbol{q} \in Q$.

Proof. The $R\left(\boldsymbol{q}_{0}\right)$ defined by the corollary of Lemma 3 constitute an open covering of $Q$. But, by Lindelöf's Theorem, ${ }^{8}$ one can select from any open covering a countable subcovering $R_{1}, R_{2}, R_{3}, \cdots$. Replacing each $R_{h}$ by $Q_{h}=R_{h} \cap\left(R_{1} \cup \ldots \cup R_{h-1}\right)^{\prime}$, we get finally a partition of $Q$ into countably many Borel sets $Q_{h}$, such that (12) holds for all $q \in Q$.

Combining Theorems 1 and 2, we get the following solution to an abstract Cauchy problem corresponding to (1) in a Fourier transform spacé.

Corollary. Let (1) be regular. Then there exists a Borel function $C(q)$, such that the associated Banach space $B(N)$ in $\Phi$ admits a $C_{0^{-}}$ semigroup, whose semi-orbits satisfy $\left(3^{*}\right)$, with $\left\|T_{t}\right\|_{N} \leqq e^{\sigma t}$ for $t \geqq 0$.

6. Differentiable functions. We now show that, for $\boldsymbol{\phi}_{0}(\boldsymbol{q})$ vanishing fast enough at infinity, the semi-orbits of $T_{t}\left[\phi_{0}\right]$ for $t \geqq 0$ define, through (4), solutions of (1) in the literal sense. ${ }^{9}$

It is well known $[2$, p. 8$]$ that $v(x)$ is defined by (4) as a continuous function, whenever $\boldsymbol{\phi}(\boldsymbol{q}) \in L_{1}$. The following result is also easily established.

LEMma 4. Let $\phi \in \Phi$ satisfy

$$
\int|\boldsymbol{\phi}(\boldsymbol{q})|\left(1+\sum\left|p_{j k}(i \boldsymbol{q})\right|\right) d Q<+\infty,
$$

${ }^{8}$ See G. T. Whyburn, Analytic topology, New York, 1942, p. 4.

9 This is closely related to some results of $[3]$ and $[4]$; see also $[2$, pp. 26, 57, 125] and $[7]$. 
where $|\boldsymbol{\phi}|=\left(\boldsymbol{\phi} \boldsymbol{\phi}^{* *}\right)^{1 / 2}$ is the ordinary norm on $E_{n}$. Then, if $\mathbf{v}(\mathbf{x})$ is defined by (4),

$$
\sum p_{j k}(\boldsymbol{D}) v_{k}(\mathbf{x})=P_{j}[\boldsymbol{v}]=\int_{\boldsymbol{Q}} \exp \left(i \boldsymbol{q} \cdot \mathbf{x} \sum p_{j k}(i \boldsymbol{q}) \phi_{k}(\boldsymbol{q}) d Q\right)
$$

exists for all real $r$-vectors $\mathrm{x}$.

Proof. By (13), the right side of (14), whose integrand is a Borel function $^{3}$ and so measurable, is Lebesgue integrable to a continuous function. The rest of the proof is an obvious extension of standard results $[2$, p. 8$]$.

The following sharper result is less easy.

Theorem 3. For all $\tau,|\tau-t|<\epsilon$, let $\boldsymbol{f}(\boldsymbol{q} ; t)$ satisfy $\left(3^{*}\right)$, and also

$$
|\boldsymbol{f}(\boldsymbol{q} ; \tau)|\left(1+\sum\left|p_{j k}(i \boldsymbol{q})\right|\right) \leqq M(\boldsymbol{q}), \quad \text { a.e., }
$$

where

$$
\int M(q) d Q<+\infty
$$

Then (1) is satisfied by the Fourier transform

$$
u(x ; t)=\int f(q ; t) e^{i q \cdot x} d Q .
$$

Proof. By Lemma 4, the right side of (1) is defined as a continuous function in space, throughout $|\tau-t|<\epsilon$. Therefore, if $|\Delta t|<\epsilon$, the difference quotient $\Delta u / \Delta t$ is defined. Hence, if (1) failed, we could find an $\mathrm{x}$ and a sequence of $\Delta t_{m} \rightarrow 0$, such that

$$
(\Delta \mathbf{u} / \Delta t)_{m}=\left[\boldsymbol{u}\left(\mathbf{x} ; t+\Delta t_{m}\right)-\boldsymbol{u}(\mathbf{x} ; t)\right] / \Delta t_{m}
$$

failed to converge to $P[u(x ; t)]$, as defined by (1). We shall now show that this is impossible, which will complete the proof. Indeed, for each $x$

$$
\begin{aligned}
\frac{\Delta \boldsymbol{u}}{\Delta t_{m}} & =\frac{1}{\Delta t_{m}} \int e^{i \boldsymbol{q} \cdot \mathbf{x}}\left[\boldsymbol{f}\left(\boldsymbol{q} ; t+\Delta t_{m}\right)-\boldsymbol{f}(\boldsymbol{q} ; t)\right] d Q \\
& =\int e^{i \boldsymbol{q} \cdot \mathbf{x}} d Q\left\{\frac{1}{\Delta t_{m}} \int_{t}^{t+\Delta t_{m}} P[\boldsymbol{f}(\boldsymbol{q} ; \tau)] d \tau\right\}
\end{aligned}
$$

where $\boldsymbol{P}[\boldsymbol{f}]$ is defined by $P_{j}[\boldsymbol{f}]=\sum p_{j k}(i \boldsymbol{q}) f_{k}$. Hence

$$
\frac{\Delta u}{\Delta t_{m}}-P[u]=\int_{Q} e^{i \boldsymbol{q} \cdot \mathbf{x}} d Q\left\{\frac{1}{\Delta t_{m}} \int_{t}^{t+\Delta t_{m}} P[\boldsymbol{f}(\boldsymbol{q} ; \tau)-\boldsymbol{f}(\boldsymbol{q} ; t)] d \tau\right\}
$$


in the sense of iterated Lebesgue integration. Since $\boldsymbol{P}$ is a matrix independent of $t$, while $\boldsymbol{f}(\boldsymbol{q} ; t)$ depends continuously on $t$, it is clear that, for each fixed $q$,

$$
\frac{1}{\Delta t_{m}} \int_{t}^{t+\Delta t_{m}} P[\boldsymbol{f}(\boldsymbol{q} ; \tau)-\boldsymbol{f}(\boldsymbol{q} ; t)] d \tau=\boldsymbol{\Delta}_{m}(\boldsymbol{q}) \rightarrow 0 \text { as } \Delta t_{m} \rightarrow 0 .
$$

Hence, by Lebesgue's Dominated Convergence Theorem and (16), $\Delta u / \Delta t_{m} \rightarrow P[u]$ for each fixed $x$ provided

$$
\left|\boldsymbol{\Delta}_{m}(\boldsymbol{q})\right| \leqq K(\boldsymbol{q}), \quad \int_{Q} K(\boldsymbol{q}) d Q<+\infty .
$$

But, by definition,

$$
\left|\Delta t_{m} \boldsymbol{\Delta}_{m}\right|^{2}=\sum_{j=1}^{n}\left|\int_{t}{ }^{t+\Delta t_{m}} \sum_{k=1}^{n} p_{j k}(i \boldsymbol{q})\left[f_{k}(\boldsymbol{q}, \boldsymbol{\tau})-f_{k}(\boldsymbol{q} ; t)\right] d \tau\right|^{2} .
$$

Applying Schwarz' Inequality, we get

$$
\begin{aligned}
\left|\Delta t_{m} \Delta_{m}\right| & \leqq \sum_{j=1}^{m} \int_{t}^{t+\Delta t_{m}}\left(\sum\left|p_{j k}\right|^{2}\right)^{1 / 2}(|\boldsymbol{f}(\tau)|+|\boldsymbol{f}(t)|) d \tau \\
& \leqq 2 M(q) \Delta t_{m} \quad \text { by }\left(13^{*}\right) .
\end{aligned}
$$

This gives (17), with $K(\boldsymbol{q})=2 M(\boldsymbol{q})$.

7. Concrete Cauchy problem. Suitably combining Theorem 3 with the methods used in proving Theorems $1-2$, we can also solve the concrete Cauchy problem. First, we observe that, in $\$ 4$, the results are unchanged if we replace $C(\boldsymbol{q})$ by $w(\boldsymbol{q}) C(\boldsymbol{q})$ and $C\left(\boldsymbol{q}_{0}\right)$ by $w\left(\boldsymbol{q}_{0}\right) C\left(\boldsymbol{q}_{0}\right)$, for any positive scalars $w(\boldsymbol{q})$ and $w\left(\boldsymbol{q}_{0}\right)$. In particular, the modulus of the $T_{t}(\boldsymbol{q})$ is unchanged, while $N(\boldsymbol{q} ; \boldsymbol{\phi})$ is replaced by $N_{w}(\boldsymbol{q} ; \boldsymbol{\phi})$ $=w(\boldsymbol{q}) N(\boldsymbol{q} ; \boldsymbol{\phi})$.

By choosing the $w(\boldsymbol{q})$ sufficiently large, however, we can make semi-orbits in the complex Banach space $B\left(N_{w}\right)$ of the $\boldsymbol{\phi}(\boldsymbol{q}) \in \Phi$ such that (cf. (8))

$$
\int N(\boldsymbol{q} ; \boldsymbol{\phi}(\boldsymbol{q}) w(\boldsymbol{q}) d Q<+\infty,
$$

consist entirely of functions which satisfy the conditions of $\S 6$.

More precisely, any Borel function $C(\boldsymbol{q})$ of the Corollary to Theorem 2 can be modified in the following way. For $q \in Q, q$ is in some $Q_{h}$ and $C(\boldsymbol{q})=C\left(\boldsymbol{q}_{h}\right)$ where $Q_{h} \subseteq R_{h}=R\left(\boldsymbol{q}_{h}\right)$. Let $w(\boldsymbol{q})=w\left(\boldsymbol{q}_{h}\right)$ where $w\left(\boldsymbol{q}_{h}\right)>0$ is so chosen that in $R\left(\boldsymbol{q}_{h}\right)$ 


$$
w\left(\boldsymbol{q}_{h}\right) N\left(q_{h} ; T_{t}(q)[\boldsymbol{\phi}]\right) \geqq\left(1+\sum\left|p_{j k}(i \boldsymbol{q})\right|\right) \cdot|\boldsymbol{\phi}| .
$$

Then $w(q)$ is a Borel function since it is constant on each Borel set $Q_{h}$, and $w(\boldsymbol{q}) C(\boldsymbol{q})$ defines a Banach space $B\left(N_{w}\right)$ as before. The modulus of the semigroup (5) is given by

$$
\left\|T_{t}(q)\right\| N_{w} \leqq e^{\sigma t},
$$

where

$$
N_{w}(\boldsymbol{q} ; \boldsymbol{\phi})=w(\boldsymbol{q}) N(\boldsymbol{q} ; \boldsymbol{\phi}) .
$$

It will follow that, on any interval $|t-\tau|<\epsilon$ of any semi-orbit in $B\left(N_{w}\right)$, the hypotheses of Theorem 3 will be satisfied, with $M(\boldsymbol{q})$ $=\sup _{\tau} e^{\sigma \tau} N_{w}(\boldsymbol{q} ; \boldsymbol{\phi}(\boldsymbol{q}))$. This proves

THEOREM 4. For suitable $B(N)$, every semi-orbit in the corollary of Theorem 2 represents an actual solution of (1).

We now define the system (1) to be hyperbolic if and only if $P$ and $-P$ are both regular (see $[1, \S 9]$ for a discussion of alternative definitions). Since the substitution of $-P$ for $P$ leaves the modified Jordan canonical form of $\$ 4$ unchanged except for sign, (10) holds with the substitution of $|t|$ for $t$. This proves the

COROLlaRY. If (1) is hyperbolic, then the $C_{0}$-semigroups of Theorem 4 and Corollary of Theorem 2 are parts of $C_{0^{-}}$-groups, with moduli $\left\|T_{t}\right\|$ $\leqq e^{\sigma|t|}$.

\section{BIBLIOGRAPHY}

1. G. Birkhoff, Journal of the Society for Industrial and Applied Mathematics vol. 2 (1954) pp. 57-67.

2. S. Bochner and K. Chandrasekharan, Fourier transforms, Princeton, 1949.

3. I. Gelfand and G. Silov, Uspehi Matematičeskih Nauk. vol. 6 (1953) pp. 3-54.

4. - J. Math. Pure Appl. vol. 4 (1956) pp. 383-413.

5. E. Hille, Functional analysis and semigroups, Amer. Math. Soc. Colloquium Publications, vol. 31, 1948.

6. R. S. Phillips, Bull. Amer. Math. Soc. vol. 61 (1955) pp. 16-33.

7. L. Schwartz, Annales de l'Institut Fourier vol. 2 (1950) pp. 19-49.

HARVARD UNIVERSITY 